\title{
Business Process Modeling Based on Norm
}

\author{
Jun Zhao", Xixia Wang ${ }^{2}$ \\ ${ }^{1}$ School of Economics and Management, Ningxia University, Yinchuan, China; ${ }^{2}$ School of Mathematical and Computer Sciences, \\ Ningxia University, Yinchuan, China. \\ Email: \{wwwzhaojun, 148112319\}@163.com
}

Received August 25 ${ }^{\text {th }}$, 2011; revised September 22 ${ }^{\text {nd }}, 2011$; accepted November $2^{\text {nd }}, 2011$.

\begin{abstract}
As to the question that the current enterprise information system can not adapt quickly to shifting business, this paper mainly studied business process adaptability according to Norm and SOA and proposed a business process modeling method based on Norm. The purpose is to enable business change in the information systems more flexible and better meet the current market and customer demands.
\end{abstract}

Keywords: Norm, Business Process Modeling, UML

\section{Introduction}

With the rapid development of economic globalization, the enterprise market and customer requirement are changing rapidly. Accordingly, the enterprise business process has also shown rapidly change, dynamic and diverse features. As is well known, business process is the core of enterprise operation and plays an important role. When the enterprise business processes frequently change, the existing information system may need to make large changes, even need to build a new information system. Resulting in enterprise information systems can not adapt quickly to business requirement of enterprises. This has seriously affected the function of enterprise information systems and restricted the development of enterprises.

Under this background, this paper studies business process adaptability of information system. The purpose is to enable business change in the information systems more flexible and better meet the current market and customer requirement [1].

\section{Research on Business Processes Adaptability}

The study on business process adaptability is currently focused on three areas [2-4]. First, it is from the implementation technique perspective, how the information system to achieve these changes in business processes. Now, the main application is SOA technology. Next, it is from the software requirements analysis and business process modeling perspective, research the business process adaptability to meet the needs of process reengi- neering. Finally, consider the establishment of a flexible business process model, so that it has a good adaptability to dynamic changes of all kinds in the course of implementation.

Considering the high quality of SOA and central role of the business process in the enterprise, this paper combines the first and the second areas, research on business process modeling in the process of building service-oriented information system. Business process modeling problem focused on business process modeling method and language. Current modeling methods such as, business process diagram, IDEF series model and the event process chain etc, they lack of full attention to the organization and their role. It is difficult to describe complex business process constraints. The most popular Business Process Execution Language BPEL also difficult to graphical modeling, lack of support for business rules and other issues [5].

In this paper, through introducing the Norm to analyze and express the business needs in the process of the business process modeling, a business process modeling method based on norm was proposed. The purpose is to enable information systems in business process modeling stage to be able to fully express their business requirement, eliminate ambiguity, making the software system more flexible to adapt to business changes.

\section{Research on Norm-Based Business Process Modeling Method}

Because this paper research business process modeling in the service-oriented information system, first of all introduce the service-oriented information system analysis 
process.

\subsection{Service-Oriented Information System Analysis Process}

As shown in Figure 1: Through the realization of two parallel processes of business analysis and service design, development, you can quickly develop a system model. Thus shorten the development cycle, enhance the system reusability and agility. Specific aspects responsibilities are as follows:

\section{1) System Position}

System position is generally analyze by UML deployment diagrams. It describes the static view of the software components of a hardware node and each node runtime. Our aim is to understand the relationship between service-oriented information systems and the surrounding system. Deployment diagrams show not only the system hardware, but also the relevant system software and middleware of connect heterogeneous systems.Through the deployment diagrams, we can clearly see the relationship among the multi-systems.

2) Functional Analysis

At this stage, we can describe user requirements by

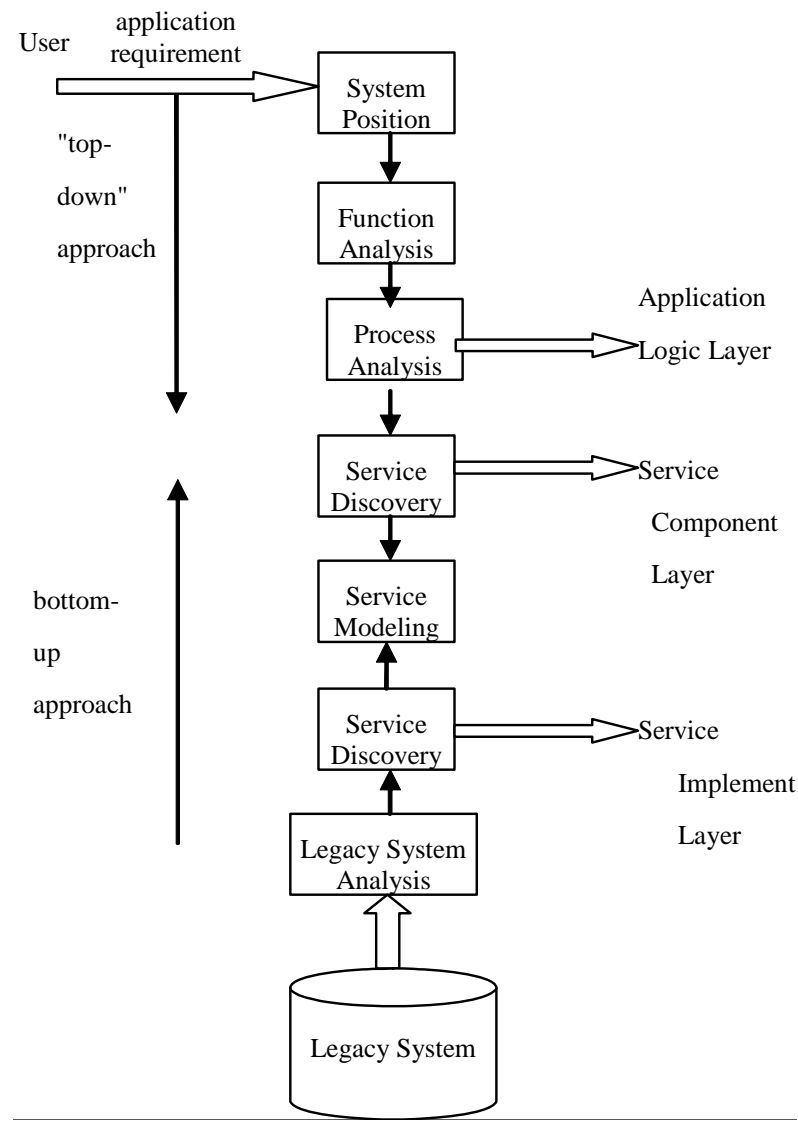

Figure 1. Service-oriented information system analysis process. use case diagram of UML. Functional analysis in the whole modeling process occupies a vital position. It affects analysis and design of other systems view. When using SOA to build a system, the system often interact with multiple heterogeneous systems. Therefore, when we analyzed the system by use case diagram, not only to analyze its own system role use case, but also to analyze the role use case of its interactive system.

3) Process Analysis

According to the analysis result of the second step by use case diagram, system functions are generally divided into two parts. One is relatively complex business process use case. It find the service operation by UML activity diagram to analyze the entire business process. The other is simple and independent use case. It can be directly as a service operation.

4) Service Discovery

According to the UML business process diagram of the third step result, the complex business processes are gradually decomposed into fine-grained processes and get the function by analysis of the activities of the processes. The function can be as service operation, with the services operation from the simple use case, you can analyze all the candidate services that systems development demands. In the development process, it must be noted that not all business functions should be achieved by service.

5) Service Modeling

The fourth step certainly knew the system contains what services and their function. Step 5 needs for standardization these services, establishment service model, implementation the mapping from abstract services to physical services.

After this step, the service modeling phase of SOAoriented basic design is completed. We finally confirmed the services and their operation that system construction requirement. Next we need to design and realize the services and their operation, development new services. That is, design and realize the interior of services and improve existing services to add manipulation.

This paper introduced the norm in the business process analysis based on the above system analysis process and proposed business process modeling method based on norm .It model business process using UML and norm.

\subsection{Norm}

Norms is an important concept in organization semilogy. In organization semiotics, the organization is essentially a system composed of social actors. In order to complete the objectives, they cooperate with each other in accordance with particular norms and constraints. So the organization can be expressed by explaining the subject, behavior and norm. The norm is generated based on the 
specific social context and describes how to interact and collaborate among different cultural backgrounds, different organizations, different departments and behavioral actor [6].

Norm is conduct guidelines in organization and be expressed by writing or informal. It coordinates and controls the behavior of the members directly or indirectly, set the communication way.

Salter divided the norm into five parts: agent details, the required information, triggers, actions can be taken, a detailed norm specification. Stamper defines the expression style of norm [7]:

Whenever <conditions set $>$ If $<$ state set $>$ Then $<$ Agent $>$ Is $<$ Deontic $>$ To $<$ action set $><$ conditions set $>$ indicates the conditions when agent perform some action; further instructions can use If <state set> that indicates the state when perform some action;

<agent> refers to the responsibility agent, it may be people or software. <deontic> including permitted, obliged and prohibition, etc. Through these moral logic operators, the active wishes of agent have been considered adequately. <Action set $>$ formulate the action set of taking in the case of conditions are met.

\subsection{Business Process Modeling Method Based on Norm}

The method extract the system analysis process of 3.1 and mainly improve it in the business layer and service layer, business process modeling.

1) Business layer business process modeling: the problem is mainly norm acquisition and representation.

At first, for the acquisition of norm, organizational semiotics provides the method of norm analysis. The process of norm analysis include: responsibility analysis, the relevant factors analysis, trigger condition analysis and detailed norm description.

Take a business process modeling for instance. Represent business processes by UML activity diagrams.

In the activity diagram, the swimming lane will group the process by role. The swimming lanes have many different uses, including prompt the place of the action or indicate the role of the action. In the activity diagram, the lane is a vertical rectangle and those activities that belong in the rectangle. The top of rectangle is the name of the corresponding organizational units or roles and activities can be placed on the implementation of the organizational unit. Through the lane, the role-based business process model and organizational model can be combined. Then insert the norm by [\#], thus the role and the corresponding norm has clearly shown in the diagram. In order to make business processes more flexible and variable, we extract some important norm that the role corresponding and realize it in the process. As a result, we can more convenient to control the change of the process.

Taking information publish system as example, the core function is the member publish information. The member publish information process diagram of based on norm as shown in Figure 2. It is based on the current process diagram. At the branch judge of activity diagram, the norm is inserted by [\#]. So that put the role and the corresponding specification in the figure has clearly shown. Moreover, according to the branch structure of the diagram, the norm of the process can be easily extracted. The norm of the business process shown in Table $\mathbf{1 .}$

2) Service layer business process modeling. There are two steps: service discovery and service modeling.

$>$ Service discovery:

Through the above business process diagram based on norm, according to the top-down decomposition method, we can get the small granularity service as the candidate process. We can also get some larger granularity services directly from the diagram and they can be further refined to achieve through atomic services composition [8].

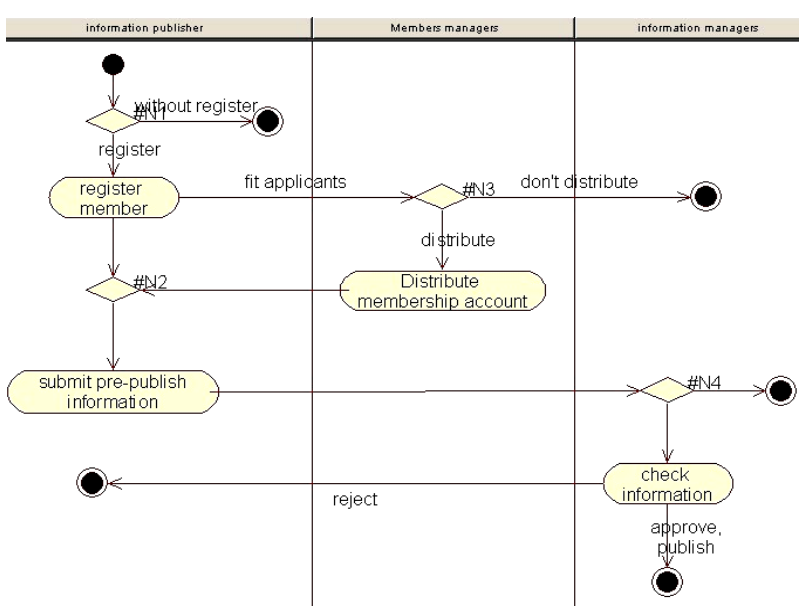

Figure 2. Business process diagrams based on norm.

Table 1. The norms of the business process.

\begin{tabular}{|c|c|}
\hline Role action & Process norms \\
\hline \multirow{4}{*}{$\begin{array}{l}\text { information } \\
\text { publish } \\
\text { process }\end{array}$} & $\begin{array}{l}\text { N1: whenever the system does not check information on } \\
\text { Saturday and Sunday. If today is Friday. Then informa- } \\
\text { tion publisher is permitted to register or no. }\end{array}$ \\
\hline & $\begin{array}{l}\text { N2: whenever submit information. If distribution ac- } \\
\text { count is successful. Then the process is permitted to } \\
\text { submit information. }\end{array}$ \\
\hline & $\begin{array}{l}\text { N3: Whenever the userquantity is full. If the user meet } \\
\text { the requirement. Then the process is prohibited to dis- } \\
\text { tribute account. }\end{array}$ \\
\hline & $\begin{array}{l}\text { N4: whenever the information of the site is insufficient. } \\
\text { If the information meet the requirement. Then informa- } \\
\text { tion manager is obliged to pass the checking. }\end{array}$ \\
\hline
\end{tabular}


a) Register service

Input parameters: user name, title, content, time, etc. Output parameters: true/false (whether registration is successful).

b) Distribution member account service

Input parameters: true/false (receive the parameter from the output parameters of the registerer service). Output parameters: true/false, ID (whether the user meet the requirements).

c) Checking information service

Input parameters: title, content and time. etc.

Output parameters: true/false (whether the information meet the requirements).

$>$ Service modeling:

At the stage of service discovery, the system conatins what services and their function were certain. Then needs for standardization these services and establishment service model, implementation the mapping from abstract services to physical services. At this stage, the main problem is how to express the norm of the business process diagram in the servcie model. Because norm is close to natural language, it is ambiguous. Here, we use the OCL to express the norm. OCL is a formal object constraint language. It can ensure the correctness and performability of the norm. This is an important step in the process of implementation information system based on norm.

Using UML to model the static structure and dynamic business process of the service as follows:

a) Static structure modeling

We describe the static part of the web service composition including their operation name and message type by UML class diagrams [9]. Web services are described by a class of a stereotype with a name "Webservice". The operation of the class described the operation that the service offers. The input and output message of the services are described by the input and output parameters of the class. The static model of the ordinary users checking service as shown in Figure 3.

b) Dynamic business process modeling

On the basis of the above static model, we extended UML activity diagram elements to define the control flow and data flow. We invoke the web service operation

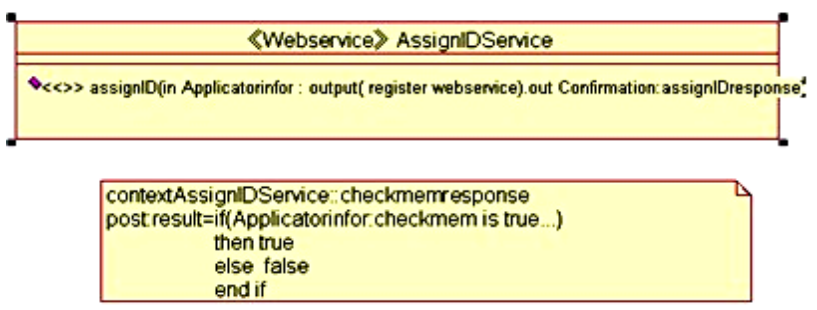

Figure 3. The static model of the ordinary user checking service. by the activity state of with the "invoke" stereotype. The tag value of the "invoke" describes the invoked web service and its prot type and operation. The operations are corresponding with the operation of the static model.

The control flow is defined by the conversion and pseudo-state (decsion, fork, join, etc.). The object flow with the stereotype "message" expresses the message transmission. A message object represents the output from one operation into the next operation. These messages correspond to the input and output messages that the static model of web services defined. The dynamic business process modeling is show on Figure 6.

\section{Case}

This section take the building supply and demand information system for agricultural as the background. It will follow the previous research part 3 process. In this process, the business process modeling method based on norm was applied and the information checking process was realized to verify the applicability and superiority of this method. The following analysis of the system step by step:

\subsection{Business Layer Business Process Modeling Based on Norm}

The core of the system processes is the members publish information process. This section selects its subprocess (checking information process) as an example to explain the model process. The Figure $\mathbf{4}$ shows the activities diagram of the checking information process.

Users need get the information application form, then complete it and submit it to the checking information process, at last obtain the results. In the Figure 2, which is shown the business process modeling based on Norm. The norms of the checking business process as shown in Table 2.

\subsection{Service Layer Business Process Modeling Based on Norm}

1) Service discovery

From the business layer analysis, the domain require-

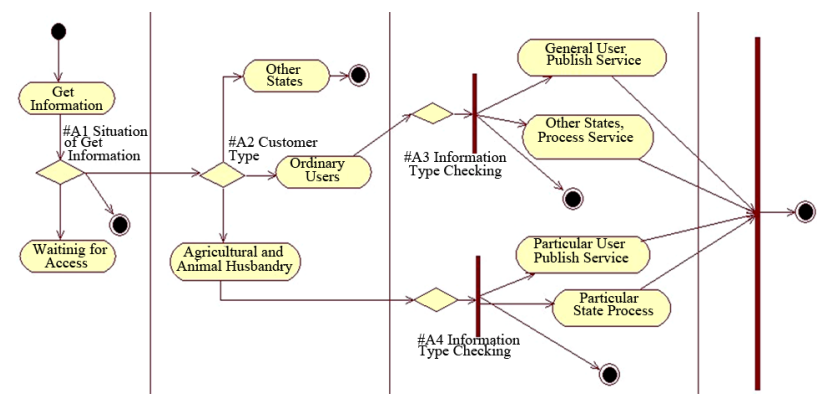

Figure 4. Checking information business process diagrams. 
Table 2. the norms of the checking business process.

\begin{tabular}{l}
$\begin{array}{c}\text { Role } \\
\text { action }\end{array}$ \\
\hline $\begin{array}{l}\text { A1: Whenever the information quantity of the site is full. If } \\
\text { application information is completely. Then checking proc- } \\
\text { ess is permitted to publish or reject. } \\
\text { Whenever the information quantity of the site is full. If } \\
\text { application information is completely. Then checking proc- } \\
\text { ess is obliged to wait for the information. } \\
\text { A2: whenever The information quantity of the site is normal. } \\
\text { If application information meet the requirement; the category } \\
\text { of user is agriculture bureau. Then checking process is } \\
\text { obliged to agriculture bureau checking service. } \\
\text { Checking Whenever the information quantity of the site is normal. If } \\
\text { process application information meet the requirement; the category } \\
\text { of user is ordinary user. Then checking process is obliged to } \\
\text { ordinary user checking service. } \\
\text { Whenever the information quantity of the site is normal. If } \\
\text { the category of user have some errors. Then checking proc- } \\
\text { ess is obliged to other cases process service. } \\
\text { A3: whenever ordinary user checking If the category of } \\
\text { information is fruit. Then checking process is obliged to } \\
\text { ordinary user publish service. } \\
\text { A4: whenever agriculture bureau checking. If the category of } \\
\text { information is Vegetables. Then checking process is obliged } \\
\text { to particular user publish service. }\end{array}$ \\
\hline
\end{tabular}

ments can better be shown in the business process based on Norm.

In the demo process, it was divided into some smaller processes. We can break out all candidate services. Here, we select a small part as follows:

a) Checking process service

Input parameters: username, title, content, time, etc.

Output parameters: true/false (Whether the information is published).

b) Ordinary user checking process service

Input parameters: username,

Output parameters: true/false (Whether the user meets the requirements).

c) Ordinary publishes service

Input parameters: title, content, time, etc.

Output parameters: true/false (Whether the information is published).

2) Building service layer business process model

The above services statically structural models and dynamic model are shown as follows.

a) Static structure model

The static model of ordinary user checking service and ordinary publishing as shown in Figure 5.

To avoid repeated, the static service model is to select to show the analysis result. In the model, Norm would be expressed by OCL in the detail. b) Service dynamic model

Fellow the previous analysis, service dynamic model as shown in Figure 6.

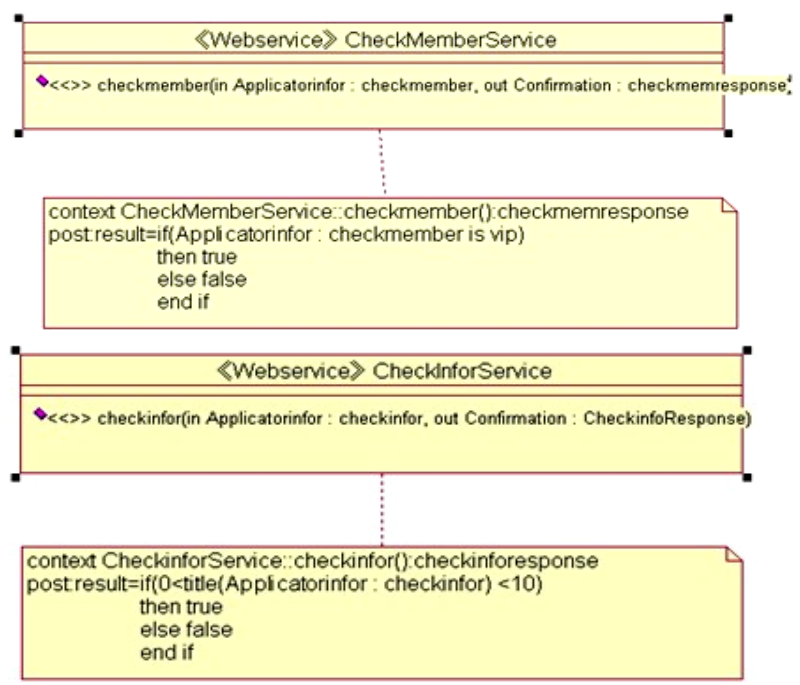

Figure 5. Service static modeling.

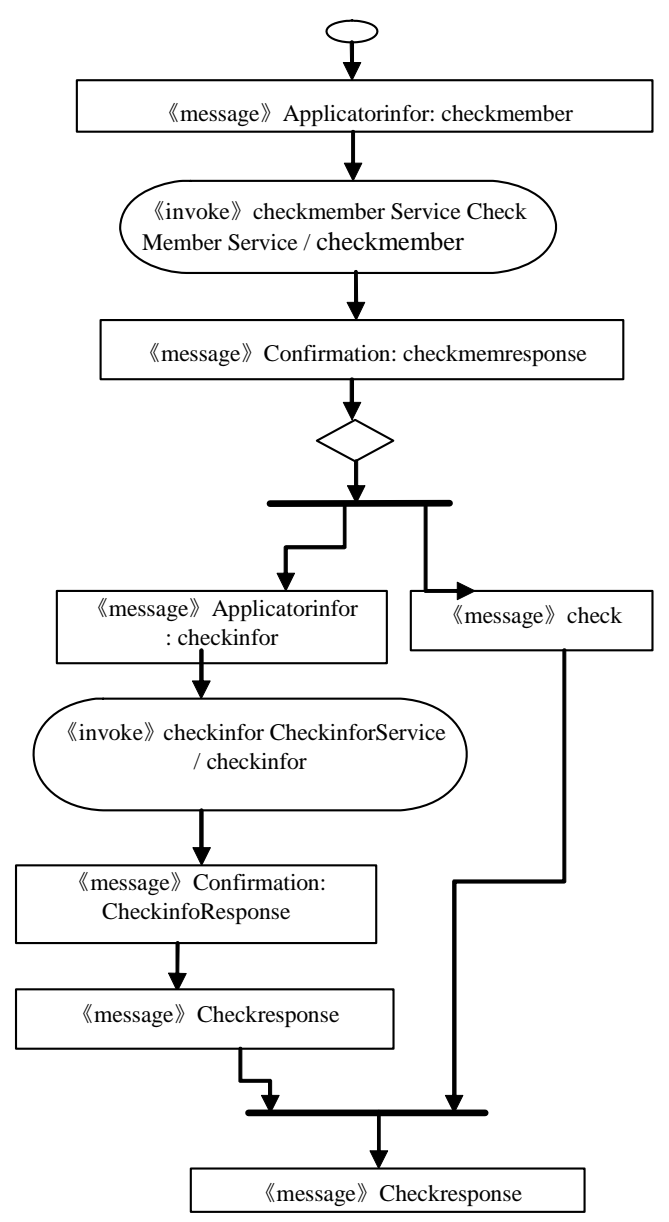

Figure 6. User checking service. 


\subsection{Implement Business Process}

To demonstrate the results of the analysis, We orchestrate the services to achieve specific business functions by BPEL software. Following the ordinary user checking information process are as an example:

1) Develop related services. User checking service; information checking service, services test page as shown Figures 7 and 8.

2) The implementation of the checking information process: If relevant services are ready, we can design the bpel processes. We can use the method of semantic web service composition to compose users' subjective services from the user requirements [10]. The process generally divided into the following steps:

- Establish partner links

- Set variables that service calling required

- BPEL process design

- Deployment and testing process

BPEL process design diagram and process test diagram are as follows Figures 9 and 10.

\section{Methods \\ - getEndpoint(0) \\ - setEndpoint (java. lang. String) \\ - getCheckmem0 \\ - checkmember (java. lang. String)}

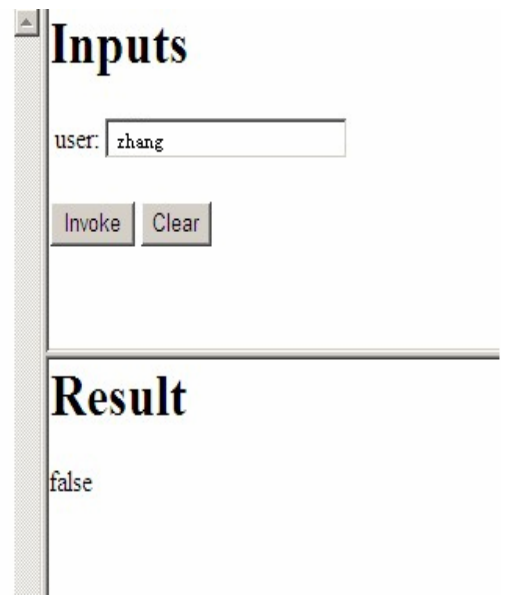

Figure 7. User checking service.

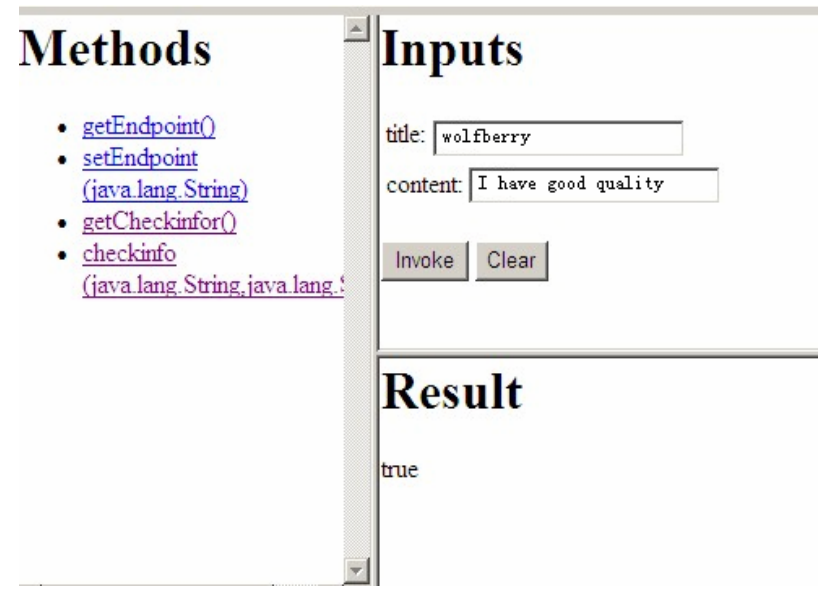

Figure 8. Information checking service.

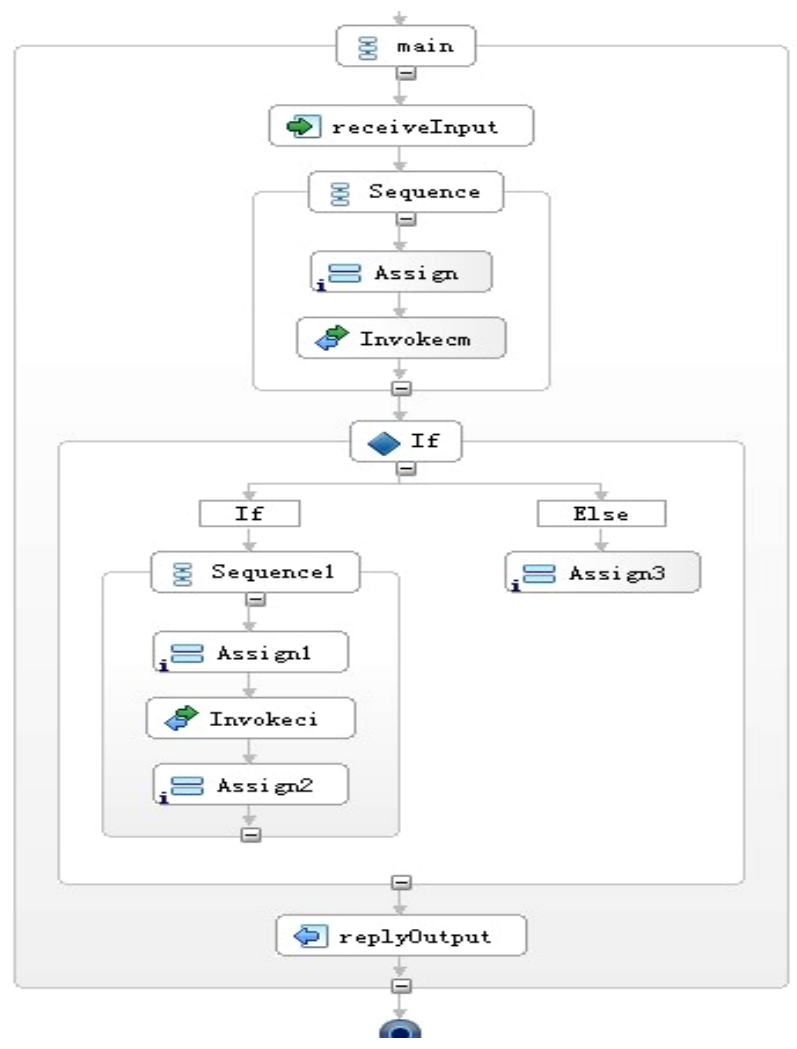

Figure 9. BPEL process diagram.

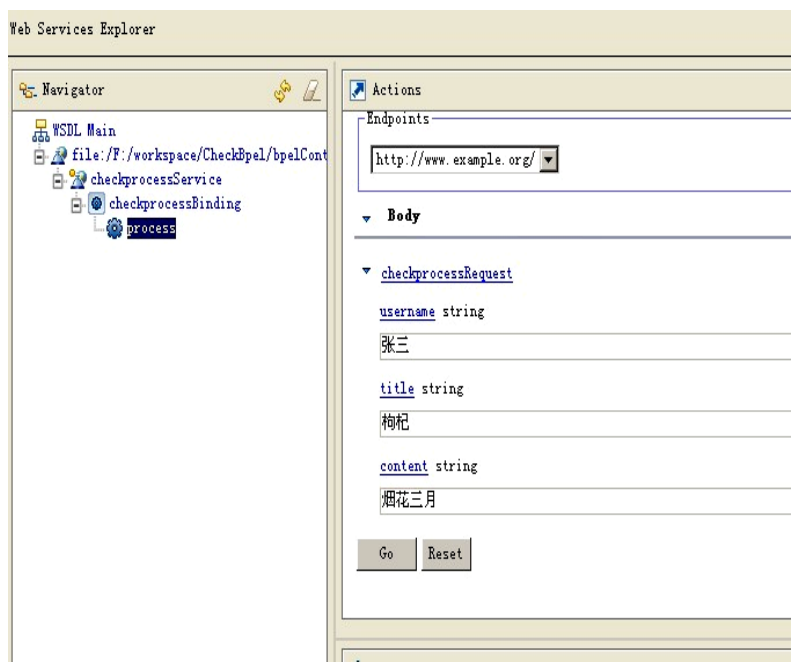

Figure 10. BPEL process test diagram.

\section{Conclusions}

This paper focus on improving enterprise information systems flexibility, adaptability and scalability and the question on business process modeling. Propose a business process modeling method based on Norm. From the business layer, we increase the analysis contents and use Norm to express for increasing the system semantic. Then use normal requires tools to translate Norm to OCL. 
Finally, we illustrate the result with BPEL tools. The research purpose is to use Norm increase the semantic of system model, and enable information systems in business process modeling stage to be able to fully express their business requirement, eliminate ambiguity, making the software system more flexible to adapt to business changes.

\section{Acknowledgements}

This research is supported by Natural Science Foundation of China (No. 70961007), the 2011 scientific and technological projects of Ningxia (Ningxia Local Government Debt Risk Early Warning and Strategy).

\section{REFERENCES}

[1] E. A. Stohr and J. L. Zhao, “A Technology Adaptation Model for Business Process Automation,” 30th Hawaii Innternational Conference on System Sciences (HICSS), Information Systems Track-Internet and the Digital Economy, Vol. 4, 1997, pp. 405.

[2] R. P. Lu and S. Z. Sadiq, "A Survey of Comparative Business Process Modeling Approaches," Lecture Notes in Computer Science, Vol. 4439, 2007, pp. 82-94. doi:10.1007/978-3-540-72035-5_7

[3] D. G. Wastell, P. White and P. Kawalek, “A Methodology for Business Process Redesign: Experiences and Issues. The Journal of Strategic Information Systems, Vol. 3, No. 1, 1994, pp. 23-40. doi:10.1016/0963-8687(94)90004-3
[4] A. McKay and Z. Radnor, "A Characterization of a Business Process," International Journal of Operations \& Production Management, Vol. 18, No. 9/10, 1998, pp. 924-936. doi:10.1108/01443579810225522

[5] D. Karastoyanova, A. Houspanossian, M. Cilia, F. Leymann and A. Buchmann, "Extending BPEL for Run Time Adaptability, edoc," 9th IEEE International EDOC Enterprise Computing Conference (EDOC'05), 2005, pp. 15-26.

[6] K. C. Liu, "Semiotics in Information Systems Engineering [M],” Cambridge: Cambridge University Press, 2000. doi:10.1017/CBO9780511543364

[7] R. Stamper, K. Liu, M. Hafkamp and Y. Ades, "Understanding the Roles of Signs and Norms in Organizations-A Semiotic Approach to Information Systems Design,” Behaviors and Information Technology, Vol. 19, No. 1, 2000, pp. 15-27. doi:10.1080/014492900118768

[8] J. Zhao and X. X. Wang, "Research on Legacy System Reuse in Ningxia Public Business Information Services Platform,” The 2010 International Conference on Computational Intelligence and Software Engineering, Wuhan, China.

[9] X. Yang, "UML Modeling Approach for Aspect-Oriented Web Services Composition," Application Research of Computers, 2008.

[10] J. Zhao and X. Z. Gu, "Applications of Norm and Situation Calculus in the Semantic Web Service Composition," Journal of Software Engineering and Applications, Vol. 3, No. 8, 2010, pp. 776-783. doi:10.4236/jsea.2010.38090 